\title{
Off-diagonal Long-Range Order and Supersolidity in a Quantum Solid with Vacancies
}

\author{
$\mathrm{Yu}$ Shi, ${ }^{1, *}$ Y Yin Yang, ${ }^{2}$ and Shao-Ming $\mathrm{Fei}^{3}$ \\ ${ }^{1}$ Department of Physics, Fudan University, Shanghai 200433, China \\ ${ }^{2}$ School of Mathematics and Statistics, Huazhong University of Science and Technology, Wuhan 430074, China \\ ${ }^{3}$ School of Mathematical Sciences, Capital Normal University, Beijing 100048, China
}

\begin{abstract}
We consider a lattice of bosonic atoms, whose number $N$ may be smaller than the number of lattice sites $M$. We study the Hartree-Fock wave function built up from localized wave functions $w(\mathbf{r})$ of single atoms, with nearest neighboring overlap. The zero-momentum particle number is expressed in terms of permanents of matrices. In one dimension, it is analytically calculated to be $\alpha N(M-N+1) / M$, with $\alpha=\left|\int w(\mathbf{r}) d \Omega\right|^{2} /[(1+2 a) l]$, where $a$ is the nearest-neighboring overlap, $l$ is the lattice constant. $\alpha$ is of the order of 1 . The result indicates that the condensate fraction is proportional to and of the same order of magnitude as that of the vacancy concentration, hence there is off-diagonal long-range order or Bose-Einstein condensation of atoms when the number of vacancies $M-N$ is a finite fraction of the number of the lattice sites $M$.
\end{abstract}

PACS numbers: 67.80.bd, 67.80.-s, 05.30.Jp

\section{INTRODUCTION}

Supersolidity refers to the superfluid-like behavior of a solid, in particular, the non-classical rotational inertia (NCRI) or missing moment of inertia, as a consequence of Bose-Einstein condensation (BEC) or off-diagonal longrange order (ODLRO) $1,2,3,4$. A few years ago, Kim and Chan observed NCRI in bulk solid ${ }^{4} \mathrm{He}$ in torsional oscillators ${ }^{-}$, which was subsequently confirmed by several other experimental groups $6,7,8,9,10$. Heat capacity exhibits a peak near the onset of NCRI ${ }^{11}$. Superfluid-like mass flow was seen close to the melting temperature ${ }^{12}$, and on melting curve $\frac{13}{}$, being carried by liquid regions at the interface $\stackrel{14}{\underline{14}}$. Recently, it has also been observed off the melting curve by injecting atoms from superfluids $\frac{15}{5}$. Increase of shear modulus was observed at low temperatures $\frac{16}{}$, but similar phenomenon in solid ${ }^{3} \mathrm{He}$ is not accompanied by NCRI, indicating that elastic stiffening alone cannot produce $\mathrm{NCRI}^{17}$.

$\mathrm{BEC}$ of zero-point vacancies in the ground state of solid Helium is the basis in some proposals of supersolidity mechanism 18,19,20. Path integral Monte Carlo studies indeed found that solid ${ }^{4} \mathrm{He}$ is commensurate without $\mathrm{BEC}^{21}$. It was argued that zero-point vacancies or interstitials are necessary for supersolidity ${ }^{22}$. Analytical calculations based on insulator-like trial wave functions also showed that a commensurate solid cannot be a supersolid $4,23,24,25$. But on the other hand, zero point vacancies are found in variational studies using Jastrow or Shadow wave function ${ }^{26}$, vacancy induced BEC was also found by using shadow wave functions 27 . A recent diffusion Monte Carlo study of commensurate solid ${ }^{4} \mathrm{He}$ using another trial wave function found a condensate fraction $\sim 10^{-4}$ and a superfluid fraction $<10^{-528}$.

Vacancy-based mechanism was disfavored by some researchers for the reasons that ${ }^{4} \mathrm{He}$ is believed to be commensurate while the vacancies tend to be phase separated because of attraction $29,30,31$. Nevertheless, the interaction between vacancies may be more complicated 32 . On the other hand, disorders such as dislocations, grain boundary and glassiness indeed appear to be impor$\operatorname{tant} 10,13,31,33,34,35$. Grain boundaries does not seem to be the fundamental origin of NCRI, which has also been observed in large crystals 36 . In considering disorder or glassiness, there are theories combining this aspect with superfluidity $37,38,39,40,41$, as well as theories without resorting to superfluidity ${ }^{42,43}$.

With all these results, the issue whether the ground state of solid ${ }^{4} \mathrm{He}$ is commensurate or incommensurate and the mechanism of NCRI in solid ${ }^{4} \mathrm{He}$ are still open questions ${ }^{44}$. It is possible that intrinsic zero point vacancy is the fundamental origin of supersolidity, while assisted by the extrinsic disorders. This possibility is consistent with the finding in simulations that the gap for vacancy creation can be closed under a moderate stress $\underline{45}$. Most recently, Anderson put forward a Gross-Pitaevskii theory of dilute gas of vacancies to account for the supersolidity, arguing that every pure Bose solid's ground state is a supersolid based on vacancies 46 .

As a theoretical approach shedding light on supersolid mechanism, it is interesting to consider phenomenological trial wave functions of a quantum solid, and examine whether they give rise to ODLRO and supersolidity. One of the trial wave functions is metal-like, which is a product of copies of the same extended single atom wave function, each being a superposition of localized wave functions at all lattice sites. This is a BEC state, even in the case of a perfect crystal. First studied in 1970s, this wave function suffers the shortcoming that the probability amplitude of a configuration with one particle on each site tends to vanish when $N \rightarrow \infty^{24}$. Recently, it was reconsidered with multiplication of Jastrow factors, which suppress multiple occupancy in a same site 47 . However, it still has the shortcoming that the equality between the lattice site and the number of atoms is a coincidence 31 . In the trial wave function used in the recent diffusion Monte Carlo study which found $\mathrm{BEC}^{28}$, the single particle part is replaced as a product of wave functions on all lattice sites, each being superposition of wave functions of all possible single occupations of this site. 
Another trial wave function is insulator-like, with the single-particle part being a symmetrized product of the localized single-atom wave functions. There is no ODLRO in such a wave function, even though there is wave function overlap between nearest neighboring atoms ${ }^{23}$. The nonexistence of ODLRO was further proved in the cases that two particles cannot come too $\operatorname{close}^{24}$, and that the sum of overlap integrals of a single atom wave function with its neighboring ones is less than unity $\underline{25}$. Recently, the nonexistence of ODLRO or NCRI was generally shown for the case that the overlap between the neighboring atoms decays exponentially or faster, with the decay constant much smaller than the system size, with or without Jastrow factors ${ }^{4}$.

On the basis of the insulator-like wave function, Imry and Schwartz introduced vacancies in the case that there is no overlap between single atom local wave functions ${ }^{24}$. They found the zero-momentum particle number to be

$$
N_{0}=N(M-N+1) \frac{\left|\int w(\mathbf{r}) d \Omega\right|^{2}}{\Omega},
$$

where $M$ is the number of total lattice sites, $N$ is the number of atoms, $w(\mathbf{r})$ is the single atom wave function, $\Omega$ is the volume. $M \approx L^{d}, \Omega \approx M l^{d}$, where $L$ is the the number of atoms on each side of the lattice, $d$ is the dimension, $l$ is the lattice constant.

But nearest neighboring overlap is crucial in a quantum solid. Moreover, there is some inconsistency in discussing BEC under the assumption that there is no overlap between neighboring atomic wave functions. If the overlap is zero, $\left|\int w(\mathbf{r}) d \Omega\right|^{2}$ also becomes zero. Then (1) becomes not useful, as $N_{0}=0$. To see this clearly, one can fiducially assume the single-atom wave function to be Gaussian, as indeed used in variational calculations of solid ${ }^{4} \mathrm{He}^{48}, \underline{49}$, i.e.

$$
w(\mathbf{r})=\frac{1}{(\sqrt{\pi} \xi)^{d / 2}} \exp \left[-\frac{1}{2}\left(\frac{r}{\xi}\right)^{2}\right],
$$

where $d$ is the dimension of the lattice. Then

$$
\left|\int w(\mathbf{r}) d \Omega\right|^{2}=(2 \sqrt{\pi} \xi)^{d} .
$$

The nearest neighboring overlap is

$$
a=\int w(\mathbf{r}) w(\mathbf{r}-\mathbf{l}) d \Omega=\exp \left[-\frac{1}{4}\left(\frac{l}{\xi}\right)^{d}\right] .
$$

Therefore the overlap $a \rightarrow 0$ means $\xi \rightarrow 0$, or $\xi \ll l$. But then $\left|\int w(\mathbf{r}) d \Omega\right|^{2} \rightarrow 0$, or $\left|\int w(\mathbf{r}) d \Omega\right|^{2} / l^{d} \ll 1$. Therefore, it is indispensable to consider nearest neighboring overlap.

In this article, we consider the Hartree-Fock wave function of a quantum solid with vacancies in presence of nearest neighboring overlap of single atom wave functions. We obtain an analytical expression for the zeromomentum particle number $N_{0}$, in terms of permanents of matrices. This expression formally reduces to Eq. (1) if the nearest neighboring overlap integral $a$ is set to be 0 . We have made the analytical calculation of $N_{0}$ in one dimension. Our result on $N_{0}$ indicates that there is ODLRO when the number of vacancies is a finite fraction of the number of lattice sites, in presence of nearest neighboring overlap between single-atom wave functions.

The rest of this article is organized as follows. In Sec. II, we consider the Hartree-Fock wave function for a quantum solid with vacancies, constructed in terms of localized single-atom wave functions. We obtain the analytical expression for the zero-momentum particle number, which is expressed in terms of the permanents of matrices. In Sec. III, we make a calculation in the case that the overlap integral between neighboring atoms is zero, reproducing the formula obtained by Imry and Schwartz. In Sec. III, we make the calculation for the case that the overlap integral is nonzero. Part of the mathematical derivation is presented in the Appendix. The summary and discussions are made in Sec. IV.

\section{TRIAL WAVE FUNCTION AND THE EXPRESSION FOR ZERO-MOMENTUM PARTICLE NUMBER IN TERMS OF PERMANENTS OF MATRICES}

We consider the following Hartree-Fock wave function of a bosonic solid with vacancies,

$$
\Psi\left(\mathbf{r}_{1} \cdots \mathbf{r}_{N}\right)=\mathcal{A} \sum_{I} \sum_{P_{I}} \prod_{i=1}^{N} w\left[\mathbf{r}_{i}-P_{I}\left(\mathbf{R}_{i}\right)\right],
$$

where $w$ is the localized single-atom wave function, which is real and nonnegative, $I$ represents a selection of $N$ sites $\left\{\mathbf{R}_{I_{1}} \cdots \mathbf{R}_{I_{N}}\right\}$ from the total $M$ sites, $P_{I}$ represents the $N$ ! permutations of these selected $N$ sites, the summation over $I$ represents $M ! / N !(M-N)$ ! different choices of the $N$ sites. The normalization constant $\mathcal{A}$ is obtained as

$$
\mathcal{A}^{-2}=\sum_{I} \sum_{I^{\prime}} \sum_{P_{I}} \sum_{P_{I^{\prime}}} \prod_{i} Q\left[P_{I}\left(\mathbf{R}_{I_{i}}\right)-P_{I^{\prime}}\left(\mathbf{R}_{I_{i}^{\prime}}\right)\right],
$$

where

$$
Q\left(\mathbf{R}-\mathbf{R}^{\prime}\right) \equiv \int w(\mathbf{r}-\mathbf{R}) w\left(\mathbf{r}-\mathbf{R}^{\prime}\right) d \Omega .
$$

In our consideration,

$$
Q\left(\mathbf{R}-\mathbf{R}^{\prime}\right)=\left\{\begin{array}{llll}
1 & : & \text { if } & \mathbf{R}-\mathbf{R}^{\prime}=0 \\
a & : & \text { if } & \left|\mathbf{R}-\mathbf{R}^{\prime}\right|=l \\
0 & : & \text { if } & \left|\mathbf{R}-\mathbf{R}^{\prime}\right|>l
\end{array}\right.
$$

$\mathcal{A}^{-2}$ can be rewritten as

$$
\mathcal{A}^{-2}=N ! \sum_{I} \sum_{I^{\prime}} \mathcal{P}\left[\Delta\left(I, I^{\prime}\right)\right],
$$

where $\mathcal{P}\left[\Delta\left(I, I^{\prime}\right)\right]$ is the permanent of an $N \times N$ submatrix $\Delta\left(I, I^{\prime}\right)$ of the $M \times M$ matrix $\mathcal{Q}$, whose elements are

$$
Q_{i j} \equiv Q\left(\mathbf{R}_{i}-\mathbf{R}_{j}\right)
$$


where $\mathbf{R}_{i}$ and $\mathbf{R}_{j}$ run over all the lattice sites. The submatrix $\Delta\left(I, I^{\prime}\right)$ is formed by choosing, from $\mathcal{Q}, N$ rows according to the set $I$ and $N$ columns according to the set $I^{\prime}$.

The permanent of an $N \times N$ matrix $\Delta$ is defined as

$$
\mathcal{P}(\Delta)=\sum_{i_{1} \cdots i_{N}} \epsilon^{i_{1} \cdots i_{N}} \Delta_{1 i_{1}} \cdots \Delta_{N i_{N}}
$$

where $\epsilon^{i_{1} \cdots i_{N}}=1$ when every two indices are different from each other, otherwise $\epsilon^{i_{1} \cdots i_{N}}=0$. In other words, a permanent is like a determinant, except that all the terms in the expansion are positive, rather than with a sign alternation.

In this article, the calculation is limited to a onedimensional lattice. The lattice sites are numbered from left to right as $1,2, \cdots, M$. $\mathcal{Q}$ is trigonal with $\mathcal{Q}_{i i}=1$, $\mathcal{Q}_{i, i+1}=\mathcal{Q}_{i+1, i}=a$, while the other elements are 0 . Therefore $\mathcal{Q}$ is

$$
\mathcal{Q}=A_{M}
$$

here we introduce a square matrix $A_{n}, n$ being a positive integer, written schematically as

$$
A_{n} \equiv\left(\begin{array}{cccccccccc}
1 & a & 0 & 0 & 0 & 0 & 0 & 0 & 0 & 0 \\
a & 1 & a & 0 & 0 & 0 & 0 & 0 & 0 & 0 \\
0 & a & 1 & a & 0 & 0 & 0 & 0 & 0 & 0 \\
0 & 0 & a & 1 & a & 0 & 0 & 0 & 0 & 0 \\
0 & 0 & 0 & a & \ddots & \ddots & 0 & 0 & 0 & 0 \\
0 & 0 & 0 & 0 & a & \ddots & \ddots & 0 & 0 & 0 \\
0 & 0 & 0 & 0 & 0 & a & 1 & a & 0 & 0 \\
0 & 0 & 0 & 0 & 0 & 0 & a & 1 & a & 0 \\
0 & 0 & 0 & 0 & 0 & 0 & 0 & a & 1 & a \\
0 & 0 & 0 & 0 & 0 & 0 & 0 & 0 & a & 1
\end{array}\right)_{n \times n}
$$

where the subscript $n \times n$ indicates that it is an $n \times n$ matrix.

For the many-body trial wave function (5), the one-particle reduced density matrix is

$$
\begin{aligned}
\rho\left(\mathbf{r}, \mathbf{r}^{\prime}\right) & =N \int \Psi\left(\mathbf{r}, \mathbf{r}_{2}, \cdots, \mathbf{r}_{N}\right) \Psi\left(\mathbf{r}^{\prime}, \mathbf{r}_{2}, \cdots, \mathbf{r}_{N}\right) d \Omega_{2} \cdots d \Omega_{N} \\
& =N \mathcal{A}^{2} \sum_{I} \sum_{I^{\prime}} \sum_{P_{I}} \sum_{P_{I^{\prime}}} w\left[\mathbf{r}-P_{I}\left(\mathbf{R}_{I_{1}}\right)\right] w\left[\mathbf{r}^{\prime}-P_{I^{\prime}}\left(\mathbf{R}_{I_{1}^{\prime}}\right)\right] \prod_{i \neq 1} Q\left[P_{I}\left(\mathbf{R}_{I_{i}}\right)-P_{I^{\prime}}\left(\mathbf{R}_{I_{i}^{\prime}}\right)\right] \\
& =\mathcal{A}^{2} N ! \sum_{I} \sum_{I^{\prime}} \sum_{i \in I, j \in I^{\prime}} w\left(\mathbf{r}-\tilde{\mathbf{R}}_{i}\right) w\left(\mathbf{r}^{\prime}-\tilde{\mathbf{R}}_{j}\right) W_{i j}
\end{aligned}
$$

where the summation over $i$ and $j$ run over the rows and columns of submatrix $\Delta\left(I, I^{\prime}\right), \tilde{\mathbf{R}}_{i} \equiv P_{I}\left(\mathbf{R}_{I_{1}}\right), \tilde{\mathbf{R}}_{j} \equiv$ $P_{I^{\prime}}\left(\mathbf{R}_{I_{1}^{\prime}}\right)$, for which $Q\left(\tilde{\mathbf{R}}_{i}-\tilde{\mathbf{R}}_{j}\right)$ is just the $(i, j)$-th element $\Delta_{i j}$ of the submatrix $\Delta\left(I, I^{\prime}\right), W_{i j}$ is the minor of $\Delta_{i j}$. Here the minor $W_{i j}$ of $\Delta_{i j}$ is defined as the permanent of the submatrix of $\Delta$ obtained by removing the $i$-th row and the $j$-th column.

The number of particles at the zero momentum state is thus

$$
\begin{aligned}
N_{0} & =\frac{1}{\Omega} \int \rho\left(\mathbf{r}, \mathbf{r}^{\prime}\right) d \Omega d \Omega^{\prime} \\
& =\frac{X_{N}(\mathcal{Q})}{Y_{N}(\mathcal{Q})} \frac{\left|\int w(\mathbf{r}) d \Omega\right|^{2}}{\Omega}
\end{aligned}
$$

where

$$
X_{N}(\mathcal{Q}) \equiv \sum_{I} \sum_{I^{\prime}} \sum_{i \in I, j \in I^{\prime}} W_{i j}
$$

$$
Y_{N}(\mathcal{Q}) \equiv \sum_{I} \sum_{I^{\prime}} \mathcal{P}\left[\Delta\left(I, I^{\prime}\right)\right] .
$$

$X_{N}(\mathcal{Q})$ is the summation of the permanants of the minors of all the elements of all the $N \times N$ submatrices $\Delta$ 's of $\mathcal{Q}$. $Y_{N}(\mathcal{Q})$ is the summation of the permanents of all the $N \times N$ submatrices $\Delta$ 's of $\mathcal{Q}$.

\section{THE CASE WITHOUT NEAREST NEIGHBORING OVERLAP}

First let us reconsider the case without overlap between neighboring single-atom wave functions, i.e. $a=0$, and show that Eq. (13) formally reduces to Eq. (11).

In this case,

$$
\mathcal{Q}=\mathcal{I}_{M}
$$


where $\mathcal{I}_{M}$ represents the $M \times M$ unit matrix. Thus in obtaining a submatrix $\Delta\left(I, I^{\prime}\right)$, once $N$ rows are chosen, there is only one choice of $N$ columns to give rise to a nonvanishing permanent. Namely, the ordering numbers, in the parent matrix $\mathcal{I}_{M}$, of the chosen columns must be equal to those of the chosen rows, i.e. $\Delta\left(I, I^{\prime}\right)$ must be a unit matrix in order to have nonvanishing permanent. Consequently,

$$
Y_{N}\left(\mathcal{I}_{M}\right)=\frac{M !}{N !(M-N) !}
$$

which is just the number of ways of choosing $N$ rows. Note that the order of the chosen rows and the order of the chosen columns both remain the same as in the parent matrix.

In order to calculate $X_{N}\left(\mathcal{I}_{M}\right)$, we need to find out all nonzero minors for all submatrices $\Delta$ 's of $\mathcal{I}_{M}$. Note that $\mathcal{P}(\Delta)=0$ does not mean $\Delta$ has no nonzero minors. Given that the parent matrix is a unit matrix $\mathcal{I}_{M}$, in order that $\Delta$ has one or more nonzero minors, $\Delta$ must be either a unit matrix $\mathcal{I}_{N}$ or diagonal with only one " 0 " diagonal element. In the former case, there are $M ! / N !(M-N)$ ! ways of making up the unit submatrix $\Delta=\mathcal{I}_{N}$, which has $N$ nonzero minors, each equal to 1 . In the latter case, one first choose $N-1$ rows and $N-1$ columns, with the same ordering numbers in the parent matrix $\mathcal{I}_{N}$, to make up $N-1$ diagonal elements " 1 ". The number of ways of doing this is $M ! /(N-1) !(M-N+1)$ !. To choose the remaining one row and one column, their ordering numbers in the parent matrix $\mathcal{I}_{N}$ must be different, such that the remaining diagonal element in $\Delta$ is " 0 ". The number of ways of doing this is $(M-N+1)(M-N)$. Each $\Delta$ so obtained only has one nonzero minor, which is equal to 1 . Therefore,

$$
\begin{aligned}
X_{N}\left(\mathcal{I}_{M}\right) & =\frac{M !}{N !(M-N) !} N+\frac{M !}{(N-1) !(M-N+1) !}(M-N)(M-N+1) \\
& =\frac{M !}{N !(M-N) !} N(M-N+1) .
\end{aligned}
$$

Substituting (16) and (17) into Eq. (13) indeed recovers Eq. (1).

\section{THE CASE WITH NEAREST NEIGHBORING OVERLAP}

With nearest neighboring overlap, the zero-momentum particle number is

$$
N_{0}=\frac{X_{N}\left(A_{M}\right)}{Y_{N}\left(A_{M}\right)} \frac{\left|\int w(\mathbf{r}) d \Omega\right|^{2}}{\Omega},
$$

where the matrix $A_{M}$ is as defined in Eq. (11).

For an arbitrary matrix $S_{m \times n}$, we introduce $Y_{k}\left(S_{m \times n}\right)$ and $X_{k}\left(S_{m \times n}\right)$, with $k \leq \min (m, n) . \quad Y_{k}\left(S_{m \times n}\right)$ is the sum of the permanents of all the $k \times k$ submatrices of $S_{m \times n} . \quad X_{k}\left(S_{m \times n}\right)$ is the sum of the permanents of all the minors of all the $k \times k$ submatrices of $S_{m \times n}$.

First, we note the existence of the relation

$$
X_{k}\left(A_{n}\right)=(n-k+1)^{2} Y_{k-1}\left(A_{n}\right)
$$

for the following reason. Every minor of a $k \times k$ submatrix of $A_{n}$ is in fact a $(k-1) \times(k-1)$ submatrix of $A_{n}$, while a $(k-1) \times(k-1)$ submatrix is a minor of many different $k \times k$ submatrices. For a given $(k-1) \times(k-1)$ submatrix of $A_{n}$, one can add an additional row and an additional column of the $A_{n}$, making up a $k \times k$ submatrix of $A_{n}$, of which the concerned $(k-1) \times(k-1)$ submatrix of $A_{n}$ is a minor. There are $(n-k+1)^{2}$ ways to do this. Hence a $(k-1) \times(k-1)$ submatrix is a minor of $(n-k+1)^{2}$ different $k \times k$ submatrices of $A_{n}$, thus we obtain the relation (19).

Therefore

$$
\frac{X_{k}\left(A_{n}\right)}{Y_{k}\left(A_{n}\right)}=\frac{(n-k+1)^{2} Y_{k-1}\left(A_{n}\right)}{Y_{k}\left(A_{n}\right)}
$$

In Appendix A, we obtain that for $n \geq 2$,

$$
\begin{aligned}
Y_{2}\left(A_{n}\right) & =(1+2 a)^{2} \frac{n(n-1)}{2}-\left(5 a^{2}+4 a\right) n+7 a^{2}+4 a \\
& =(1+2 a)^{2} \frac{n(n-1)}{2 !}\left[1+O\left(\frac{1}{n}\right)\right],
\end{aligned}
$$

where $O(1 / n)$ represents a term of the order of $1 / n$.

In Appendix $\mathrm{B}$, we obtain that for any $3 \leq k<n$,

$$
\begin{aligned}
Y_{k}\left(A_{n}\right)= & \sum_{l=k-1}^{n-1} Y_{k-1}\left(A_{l}\right)+2 \sum_{s=1}^{k-2} a^{s} \sum_{l=k-s}^{n-s-1} Y_{k-s}\left(A_{l}\right)+a^{2} \sum_{l=k-2}^{n-2} Y_{k-2}\left(A_{l}\right) \\
& +(1+2 a) 2 a^{k-1}(n-k)(n-k+1) .
\end{aligned}
$$


From this relation, we know that $Y_{k}\left(A_{n}\right)>$ $Y_{k-1}\left(A_{n-1}\right), Y_{k-1}\left(A_{n-1}\right)$ being merely one term in the first summation in RHS of (22). Consequently, in the summation over $s, 2 \sum_{l=k-s}^{n-s-1} Y_{k-s}\left(A_{l}\right)$, which also depends on $a$, by which $a^{s}$ is multiplied, decreases with the increase of $s$. Since $a<1$, RHS of (22) converges with respect to $a$. Also note that the last term is of the power of $a^{k-1}$.

Therefore, $Y_{k}\left(A_{n}\right)$ can be written as

$$
\begin{aligned}
Y_{k}\left(A_{n}\right) & =\left[\sum_{l=k-1}^{n-1} Y_{k-1}\left(A_{l}\right)+2 a \sum_{l=k-1}^{n-2} Y_{k-1}\left(A_{l}\right)\right]\left[1+O_{\leq}(a)\right] \\
& =\left[(1+2 a) \sum_{l=k-1}^{n-1} Y_{k-1}\left(A_{l}\right)-2 a Y_{k-1}\left(A_{n-1}\right)\right]\left[1+O_{\leq}(a)\right],
\end{aligned}
$$

where $O_{\leq}(a)$ denotes a term at most of the order of $a$.

In the following, we show by induction that

$$
Y_{k}\left(A_{n}\right)=(1+2 a)^{k} \frac{n !}{k !(n-k) !}\left[1+O_{\leq}(a)\right] .
$$

Suppose that the similar identity is valid for $Y_{k-1}\left(A_{l}\right)$, with $k-1 \leq l<n$, i.e.,

$$
Y_{k-1}\left(A_{l}\right)=(1+2 a)^{k-1} \frac{l !}{(k-1) !(l-k+1) !}\left[1+O_{\leq}(a)\right] .
$$

Then

$$
(1+2 a) \sum_{l=k-1}^{n-1} Y_{k-1}\left(A_{l}\right)=\frac{(1+2 a)^{k}}{(k-1) !} \sum_{l=k-1}^{n-1} \frac{l !}{(l-k+1) !}\left[1+O_{\leq}(a)\right]
$$

Using the identity 50

$$
\sum_{j=1}^{p} j(j+1) \cdots(j+q)=\frac{1}{q+2} \frac{(p+q+1) !}{(p-1) !}
$$

we obtain

$$
\sum_{l=k-1}^{n-1} \frac{l !}{(l-k+1) !}=\frac{1}{k} \frac{n !}{(n-k) !}
$$

Hence (26) becomes

$$
(1+2 a) \sum_{l=k-1}^{n-1} Y_{k-1}\left(A_{l}\right)=(1+2 a)^{k} \frac{n !}{k !(n-k) !}\left[1+O_{\leq}(a)\right] .
$$

On the other hand, according to the assumption (25) and the above result (29), we have

$$
\begin{aligned}
2 a Y_{k-1}\left(A_{n-1}\right) & =2 a(1+2 a)^{k-1} \frac{(n-1) !}{(k-1) !(n-k) !}\left[1+O_{\leq}(a)\right] \\
& =\frac{2 a}{1+2 a} \frac{k}{n}\left[(1+2 a) \sum_{l=k-1}^{n-1} Y_{k-1}\left(A_{l}\right)\right]\left[1+O_{\leq}(a)\right] \\
& =O(a) \frac{k}{n} \sum_{l=k-1}^{n-1} Y_{k-1}\left(A_{l}\right),
\end{aligned}
$$

where $k / n \leq 1$.

Substituting (29) and (30) into Eq. (23) yields Eq. (24), 
hence the proof completes.

Then we substitute the proved identity (24) into Eq. (20), obtaining

$$
\frac{X_{k}\left(A_{n}\right)}{Y_{k}\left(A_{n}\right)} \approx \frac{k(n-k+1)}{1+2 a}
$$

if $a \ll 1$.

Therefore, according to (18), the zero-momentum particle number is

$$
N_{0} \approx \frac{N(M-N+1)}{1+2 a} \frac{\left|\int w(\mathbf{r}) d \Omega\right|^{2}}{\Omega} .
$$

This identity formally reduces to that obtained by Imry and Schwartz long ago when we set $a=0$. But $\left|\int w(\mathbf{r}) d \Omega\right|^{2} \neq 0$ only if $a \neq 0$.

On the other hand, in case $n \gg k$, no matter whether $a \ll 1$, we can also obtain the identity (31) and thus the result (32). This condition does not correspond to the physical situation concerning solid ${ }^{4} \mathrm{He}$, as that would mean most of the lattice sites are empty. For completeness, we give the mathematical proof for this case in Appendix C.

\section{SUMMARY AND DISCUSSIONS}

To summarize, we have studied the Hartree-Fock wave function of a lattice of atoms with $N \leq M$, where $M$ and $N$ are the numbers of lattice sites and atoms, respectively. The Hartree-Fock wave function is constructed in terms of localized wave functions of single atoms, with nearest neighboring overlap.

In one dimension, under this wave function, we have obtained the zero-momentum particle number as given in (32), which can be rewritten as

$$
N_{0}=\alpha \frac{N(M-N+1)}{M},
$$

where

$$
\alpha=\frac{\left|\int w(\mathbf{r}) d \Omega\right|^{2}}{(1+2 a) l}
$$

is a finite fraction of the order of 1 .

To be specific, let us again use the Gaussian wave function for $w(\mathbf{r})$, as given in (2). Then in one dimension,

$$
\alpha=\frac{2 \sqrt{\pi} \frac{\xi}{l}}{\left[1+2 \exp \left(-\frac{1}{4}\left(\frac{l}{\xi}\right)^{2}\right]\right.},
$$

which is of the order of 1 when $\xi / l$ is a finite fraction around 0.36 , which is obtained from the Lindemann ratio $\delta \approx 0.29$ for solid ${ }^{4} \mathrm{He}^{49}$, using $\delta \equiv \sqrt{\left\langle r^{2}\right\rangle} / l=\sqrt{3 / 2} \xi / l$ under the Gaussion wave function (2) . For $d=3,1 /(1+$ $2 a$ ) should be replaced by another function $f(\alpha)$ of $\alpha$, which should still be of the order of 1 . Anyway, $\alpha=$ $(2 \sqrt{\pi} \xi / l)^{3} f(\alpha)$ must be of the order of 1 .

Therefore there is BEC of atoms, i.e. $N_{0}$ is a finite fraction of $N$, when the number of vacancies $M-N$ is a finite fraction of the number of lattice sites $M$. This condition also implies that the number of atoms $N$ is a finite fraction of $M$.

Interestingly, the condensate fraction $N_{0} / N$ is proportional to and of the order of vacancy concentration $(M-N) / M$,

$$
\frac{N_{0}}{N}=\alpha \frac{M-N}{M}
$$

Currently, the experimental upper bound of vacancy concentration is about $0.4 \% \underline{26}$. Hence a Hartree-Fock wave function for a solid with zero point vacancy implies that the condensate fraction is about $0.004 \alpha$, which is very reasonable.

Moreover, for such low vacancy concentration, one has

$$
\frac{N_{0}}{M-N} \approx \alpha
$$

i.e. $\alpha$ equals the number of condensed atoms per vacancy. This is well consistent with the result of variational simulation based on Shadow wave function, which gives 0.23 condensed atoms per vacancy at $54 \mathrm{bar}^{27}$.

The Hartree-Fock wave function could be the ground state of a mean field theory. Although it is not multiplied by the Jastrow factor, the double occupancy is excluded by construction. Our calculation is done for one dimension. In three dimensions, there should not be qualitative difference in order of magnitude from the result for one dimension. Hence our result is qualitatively informative for solid ${ }^{4} \mathrm{He}$, suggesting that its supersolidity based on BEC of atoms induced by zero point vacancy is possible.

\section{Acknowledgments}

We thank A. J. Leggett, X. Q. Li-Jost, L. Reatto and Y. S. Wu for useful discussions. This work is supported by National Science Foundation of China (Grant No. 10674030), Shuguang Project (Grant No. 07S402) and The Ministry of Science and Technology of China (Grant No. 2009CB929204). 
APPENDIX A: $Y_{2}\left(A_{n}\right)$

Here we calculate $Y_{2}\left(A_{n}\right)(n \geq 2)$, i.e. the sum of the permanents of all the $2 \times 2$ submatrices of $A_{n}$. In addition to the definition of $A_{n}$ as given in (11), we shall also use matrices

$$
B_{n-1} \equiv\left(\begin{array}{ccccccccc}
a & a & 0 & 0 & 0 & 0 & 0 & 0 & 0 \\
0 & 1 & a & 0 & 0 & 0 & 0 & 0 & 0 \\
0 & a & 1 & a & 0 & 0 & 0 & 0 & 0 \\
0 & 0 & a & 1 & a & 0 & 0 & 0 & 0 \\
0 & 0 & 0 & \ddots & \ddots & 0 & 0 & 0 & 0 \\
0 & 0 & 0 & 0 & \ddots & \ddots & a & 0 & 0 \\
0 & 0 & 0 & 0 & 0 & a & 1 & a & 0 \\
0 & 0 & 0 & 0 & 0 & 0 & a & 1 & a \\
0 & 0 & 0 & 0 & 0 & 0 & 0 & a & 1
\end{array}\right)_{(n-1) \times(n-1)}
$$

The permanent of an $n \times n$ matrix $S$ is equal to

$$
\mathcal{P}(S)=\sum_{j} S_{i j} W_{i j}
$$

where $W_{i j}$ is the minor of the $S_{i j}$. Using this property, we can expand $Y_{2}\left(A_{n}\right)$ as

$$
\begin{aligned}
& Y_{2}\left(A_{n}\right) \equiv Y_{2}\left(\begin{array}{cccccccccc}
1 & a & 0 & 0 & 0 & 0 & 0 & 0 & 0 & 0 \\
a & 1 & a & 0 & 0 & 0 & 0 & 0 & 0 & 0 \\
0 & a & 1 & a & 0 & 0 & 0 & 0 & 0 & 0 \\
0 & 0 & a & 1 & a & 0 & 0 & 0 & 0 & 0 \\
0 & 0 & 0 & a & \ddots & \ddots & 0 & 0 & 0 & 0 \\
0 & 0 & 0 & 0 & a & \ddots & \ddots & 0 & 0 & 0 \\
0 & 0 & 0 & 0 & 0 & a & 1 & a & 0 & 0 \\
0 & 0 & 0 & 0 & 0 & 0 & a & 1 & a & 0 \\
0 & 0 & 0 & 0 & 0 & 0 & 0 & a & 1 & a \\
0 & 0 & 0 & 0 & 0 & 0 & 0 & 0 & a & 1
\end{array}\right)_{n \times n} \\
&= Y_{1}\left(A_{n-1}\right)+a Y_{1}\left(B_{(n-1) \times(n-1)}\right)+a Y_{1}\left(C_{(n-2) \times(n-1)}\right)+Y_{2}\left(A_{n-1}\right) \\
&=Y_{2}\left(A_{n-1}\right)+Y_{1}\left(A_{n-1}\right)+2 a Y_{1}\left(A_{n-2}\right)+3 a^{2},
\end{aligned}
$$

where we have used $Y_{1}\left(B_{(n-1) \times(n-1)}\right)=2 a+Y_{1}\left(A_{n-2}\right), Y_{1}\left(C_{(n-2) \times(n-1)}\right)=a+Y_{1}\left(A_{n-2}\right)$, which are straightforward. In this way, we obtain the following set of identities

$$
\begin{aligned}
Y_{2}\left(A_{n}\right)-Y_{2}\left(A_{n-1}\right) & =Y_{1}\left(A_{n-1}\right)+2 a Y_{1}\left(A_{(n-2)}\right)+3 a^{2}, \\
Y_{2}\left(A_{n-1}\right)-Y_{2}\left(A_{n-2}\right) & =Y_{1}\left(A_{n-2}\right)+2 a Y_{1}\left(A_{(n-3)}\right)+3 a^{2}, \\
& \vdots \\
Y_{2}\left(A_{3}\right)-Y_{2}\left(A_{2}\right) & =Y_{1}\left(A_{2}\right)+2 a Y_{1}\left(A_{1}\right)+3 a^{2} .
\end{aligned}
$$

Adding these identities together gives rise to

$$
Y_{2}\left(A_{n}\right)-Y_{2}\left(A_{2}\right)=Y_{1}\left(A_{n-1}\right)+(1+2 a) \sum_{j=2}^{n-2} Y_{1}\left(A_{j}\right)+2 a Y_{1}\left(A_{1}\right)+3 a^{2}(n-2) .
$$


Clearly $Y_{2}\left(A_{2}\right)=1+a^{2}, Y_{1}\left(A_{j}\right)=j+2(j-1) a$. Hence it can be obtained that

$$
Y_{2}\left(A_{n}\right)=(1+2 a)^{2} \frac{n(n-1)}{2}-\left(5 a^{2}+4 a\right) n+7 a^{2}+4 a,
$$

which is also satisfied when $n=2$, as $Y_{2}\left(A_{2}\right)=1+a^{2}$.

\section{APPENDIX B: $Y_{k}\left(A_{n}\right)$}

We now calculate $Y_{k}\left(A_{n}\right)$ for $3 \leq k<n$, in a way similar to the calculation of $Y_{2}\left(A_{n}\right)$ above.

Similar to (A4), we obtain

$$
\begin{aligned}
& Y_{k}\left(A_{n}\right) \equiv Y_{k}\left(\begin{array}{cccccccccc}
1 & a & 0 & 0 & 0 & 0 & 0 & 0 & 0 & 0 \\
a & 1 & a & 0 & 0 & 0 & 0 & 0 & 0 & 0 \\
0 & a & 1 & a & 0 & 0 & 0 & 0 & 0 & 0 \\
0 & 0 & a & 1 & a & 0 & 0 & 0 & 0 & 0 \\
0 & 0 & 0 & a & \ddots & \ddots & 0 & 0 & 0 & 0 \\
0 & 0 & 0 & 0 & a & \ddots & \ddots & 0 & 0 & 0 \\
0 & 0 & 0 & 0 & 0 & a & 1 & a & 0 & 0 \\
0 & 0 & 0 & 0 & 0 & 0 & a & 1 & a & 0 \\
0 & 0 & 0 & 0 & 0 & 0 & 0 & a & 1 & a \\
0 & 0 & 0 & 0 & 0 & 0 & 0 & 0 & a & 1
\end{array}\right)_{n \times n} \\
& =Y_{k-1}\left(A_{n-1}\right)+a Y_{k-1}\left(B_{(n-1) \times(n-1)}\right)+a Y_{k-1}\left(C_{(n-2) \times(n-1)}\right)+Y_{k}\left(A_{n-1}\right) .
\end{aligned}
$$

Expansion of $Y_{k-1}\left(B_{(n-1) \times(n-1)}\right)$ gives

$$
Y_{k-1}\left(B_{(n-1) \times(n-1)}\right)=a Y_{k-2}\left(A_{k-2}\right)+Y_{k-1}\left(C_{(n-1) \times(n-2)}^{T}\right) .
$$

For any matrix $S, Y_{k-1}\left(S^{T}\right)=Y_{k-1}(S)$. Hence we have

$$
Y_{k}\left(A_{n}\right)=Y_{k}\left(A_{n-1}\right)+Y_{k-1}\left(A_{n-1}\right)+a^{2} Y_{k-2}\left(A_{n-2}\right)+2 a Y_{k-1}\left(C_{(n-2) \times(n-1)}\right) .
$$

Iterative expansion of $Y_{k-1}\left(C_{(n-2) \times(n-1)}\right)$ yields

$$
\begin{aligned}
Y_{k-1}\left(C_{(n-2) \times(n-1)}\right) & =Y_{k-1}\left(A_{n-2}\right)+a Y_{k-2}\left(C_{(n-3) \times(n-2)}\right) \\
& =\cdots \\
& =\sum_{s=1}^{k-2} a^{s-1} Y_{k-s}\left(A_{n-s-1}\right)+a^{k-2} Y_{1}\left(C_{(n-k) \times(n-k+1)}\right),
\end{aligned}
$$

where $Y_{1}\left(C_{(n-k) \times(n-k+1)}\right)=(n-k)+a+2(n-k-1) a$.

Therefore

$$
\begin{aligned}
Y_{k}\left(A_{n}\right)-Y_{k}\left(A_{n-1}\right)= & Y_{k-1}\left(A_{n-1}\right)+a^{2} Y_{k-2}\left(A_{n-2}\right)+2 a Y_{k-1}\left(C_{(n-2) \times(n-1)}\right) \\
= & Y_{k-1}\left(A_{n-1}\right)+a^{2} Y_{k-2}\left(A_{n-2}\right) \\
& +2 \sum_{s=1}^{k-2} a^{s} Y_{k-s}\left(A_{n-s-1}\right)+2 a^{k-1}[(n-k)+a+2(n-k-1) a] .
\end{aligned}
$$


Hence

$$
\begin{aligned}
Y_{k}\left(A_{n}\right)-Y_{k}\left(A_{n-1}\right)= & Y_{k-1}\left(A_{n-1}\right)+a^{2} Y_{k-2}\left(A_{n-2}\right) \\
& +2 \sum_{s=1}^{k-2} a^{s} Y_{k-s}\left(A_{n-s-1}\right)+2 a^{k-1}[a+(n-k)+2(n-k-1) a] \\
Y_{k}\left(A_{n-1}\right)-Y_{k}\left(A_{n-2}\right)= & Y_{k-1}\left(A_{n-2}\right)+a^{2} Y_{k-2}\left(A_{n-3}\right) \\
& +2 \sum_{s=1}^{k-2} a^{s} Y_{k-s}\left(A_{n-s-2}\right)+2 a^{k-1}[a+(n-1-k)+2(n-k-2) a] \\
\vdots & \\
Y_{k}\left(A_{k+2}\right)-Y_{k}\left(A_{k+1}\right)= & Y_{k-1}\left(A_{k+1}\right)+a^{2} Y_{k-2}\left(A_{k}\right) \\
& \left.+2 \sum_{s=1}^{k-2} a^{s} Y_{k-s}\left(A_{k+1-s}\right)+2 a^{k-1}[a+2+2 a]\right\} \\
Y_{k}\left(A_{k+1}\right)-Y_{k}\left(A_{k}\right)= & Y_{k-1}\left(A_{k}\right)+a^{2} Y_{k-2}\left(A_{k-1}\right) \\
& \left.+2 \sum_{i=1}^{k-2} a^{s} Y_{k-s}\left(A_{k-s}\right)+2 a^{k-1}[a+1]\right\}
\end{aligned}
$$

Adding these identities together leads to

$$
\begin{aligned}
Y_{k}\left(A_{n}\right)-Y_{k}\left(A_{k}\right) & =\sum_{l=k}^{n-1} Y_{k-1}\left(A_{l}\right)+a^{2} \sum_{l=k-1}^{n-2} Y_{k-2}\left(A_{l}\right)+2 \sum_{s=1}^{k-2} a^{s} \sum_{l=k-s}^{n-s-1} Y_{k-s}\left(A_{l}\right) \\
& +2 a^{k-1} \sum_{s=0}^{n-k-1}[a+(n-k-s)+2(n-k-1-s) a]
\end{aligned}
$$

Since $Y_{m}\left(A_{n}\right)$ exists only when $m \leq n$, we have

$$
Y_{k}\left(A_{k}\right)=Y_{k-1}\left(A_{k-1}\right)+a^{2} Y_{k-2}\left(A_{k-2}\right) .
$$

Therefore

$$
\begin{aligned}
Y_{k}\left(A_{n}\right)= & \sum_{l=k-1}^{n-1} Y_{k-1}\left(A_{l}\right)+2 \sum_{s=1}^{k-2} a^{s} \sum_{l=k-s}^{n-s-1} Y_{k-s}\left(A_{l}\right)+a^{2} \sum_{l=k-2}^{n-2} Y_{k-2}\left(A_{l}\right) \\
& +(1+2 a) 2 a^{k-1}(n-k)(n-k+1) .
\end{aligned}
$$

\section{APPENDIX C: CALCULATION OF $N_{0}$ IN THE CASE OF $n \gg k$}

Here we show that the identity (31) and thus the result (32) are also valid if $n \gg k$, no matter whether $a \ll 1$ or not. Mathematically, $n \gg k$ means $n \rightarrow \infty$ while $k$ remains finite.

In the following, we show by induction that

$$
Y_{k}\left(A_{n}\right)=(1+2 a)^{k} \frac{n !}{k !(n-k) !}\left[1+O(a) O\left(\frac{1}{n}\right)\right] .
$$

Suppose that the similar identity is valid for $Y_{k-s}\left(A_{l}\right)$, with $s \geq 1$ and $k-s \leq l<n$, i.e.,

$$
Y_{k-s}\left(A_{l}\right)=(1+2 a)^{k-s} \frac{l !}{(k-s) !(l-k+s) !}\left[1+O(a) O\left(\frac{1}{l}\right)\right] .
$$

In the second term in the exact identity $(\overline{\mathrm{B} 9})$ for $Y_{k}\left(A_{n}\right), 2 a^{s}$ is multiplied by $\sum_{l=k-s}^{n-s-1} Y_{k-s}\left(A_{l}\right)$, which can be evaluated by using the assumption (C2) to be

$$
\sum_{l=k-s}^{n-s-1} Y_{k-s}\left(A_{l}\right)=\frac{(1+2 a)^{k-s}}{(k-s) !} \sum_{l=k-s}^{n-s-1} \frac{l !}{(l-k+s) !}\left[1+O(a) O\left(\frac{1}{l}\right)\right]
$$




$$
\begin{aligned}
& =\frac{(1+2 a)^{k-s}}{(k-s+1) !} \frac{(n-s) !}{(n-k-1) !} \\
& =\left[\sum_{l=k-1}^{n-2} Y_{k-1}\left(A_{l}\right)\right] \frac{(k-2) \cdots(k-s+1)}{(1+2 a)^{s-1}} \frac{1}{(n-1) \cdots(n-s+1)},
\end{aligned}
$$

where we have used the identity (27).

In the third term in ( $(\overline{\mathrm{B} 9}), 2 a^{2}$ is multiplied by $\sum_{l=k-2}^{n-2} Y_{k-2}\left(A_{l}\right)$, which can be similarly evaluated to be

$$
\begin{aligned}
\sum_{l=k-2}^{n-2} Y_{k-2}\left(A_{l}\right) & =\frac{(1+2 a)^{k-2}}{(k-2) !} \sum_{l=k-2}^{n-2} \frac{l !}{(l-k+2) !}\left[1+O(a) O\left(\frac{1}{l}\right)\right] \\
& =\frac{(1+2 a)^{k-2}}{(k-1) !} \frac{(n-1) !}{(n-k) !} \\
& =\left[\sum_{l=k-1}^{n-2} Y_{k-1}\left(A_{l}\right)\right] \frac{k}{(1+2 a)} \frac{1}{(n-k)},
\end{aligned}
$$

where we have also used the identity (27).

Besides, the last term in (B9) is $O\left(a^{k-1}\right) O\left(n^{2}\right) \ll Y_{k-1}\left(A_{n-1}\right)=O\left(n^{k}\right)$ if $k \ll n$.

Therefore

$$
Y_{k}\left(A_{n}\right)=\left[\sum_{l=k-1}^{n-1} Y_{k-1}\left(A_{l}\right)+2 a \sum_{l=k-1}^{n-2} Y_{k-1}\left(A_{l}\right)\right]\left[1+O(a) O\left(\frac{1}{n}\right)\right],
$$

where $O(1 / n) \ll 1, O(a)$ is of the order of $a$, which we do not need to specify.

Using the assumption (C2) for $s=1$, we obtain

$$
\begin{aligned}
(1+2 a) \sum_{l=k-1}^{n-1} Y_{k-1}\left(A_{l}\right) & =(1+2 a)^{k} \sum_{l=k-1}^{n-1} \frac{l !}{(k-1) !(l-k+1) !}\left[1+O\left(\frac{1}{l}\right)\right] \\
& =\frac{(1+2 a)^{k}}{(k-1) !}[F+O(G)],
\end{aligned}
$$

where

$$
\begin{aligned}
& F=\sum_{l=k-1}^{n-1} \frac{l !}{(l-k+1) !} \\
& G=\sum_{l=k-1}^{n-1} \frac{(l-1) !}{(l-k+1) !}
\end{aligned}
$$

Using the identity (27), we obtain

$$
\begin{gathered}
F=\frac{1}{k} \frac{n !}{(n-k) !} \\
G=\frac{1}{k-1} \frac{(n-1) !}{(n-k) !} .
\end{gathered}
$$

Hence (C6) becomes

$$
(1+2 a) \sum_{l=k-1}^{n-1} Y_{k-1}\left(A_{l}\right)=\frac{(1+2 a)^{k}}{k !} \frac{n !}{(n-k) !}\left[1+O(a) O\left(\frac{1}{n}\right)\right] .
$$


On the other hand, according to (C2) and (C11),

$$
\begin{aligned}
2 a Y_{k-1}\left(A_{n-1}\right) & =\frac{2 a(1+2 a)^{k-1}}{(k-1) !} \frac{(n-1) !}{(n-k) !}\left[1+O(a) O\left(\frac{1}{n}\right)\right] \\
& =\frac{2 a}{1+2 a} \frac{k}{n}\left[(1+2 a) \sum_{l=k-1}^{n-1} Y_{k-1}\left(A_{l}\right)\right] \\
& =\left[(1+2 a) \sum_{l=k-1}^{n-1} Y_{k-1}\left(A_{l}\right)\right] O(a) O\left(\frac{1}{n}\right),
\end{aligned}
$$

for the reason that $k \ll n$.

Therefore,

$$
\begin{aligned}
& \sum_{l=k-1}^{n-1} Y_{k-1}\left(A_{l}\right)+2 a \sum_{l=k-1}^{n-2} Y_{k-1}\left(A_{l}\right) \\
= & (1+2 a) \sum_{l=k-1}^{n-1} Y_{k-1}\left(A_{l}\right)-2 a Y_{k-1}\left(A_{n-1}\right) \\
= & (1+2 a) \sum_{l=k-1}^{n-1} Y_{k-1}\left(A_{l}\right)\left[1+O(a) O\left(\frac{1}{n}\right)\right] .
\end{aligned}
$$

By using (C11) and (C13), (C5) leads to the identity (C1), which is thus proved. Substituting this proved identity into Eq. (20), we obtain Eq. (31) and thus also (32).

* Email address: yushi@fudan.edu.cn

1 A. J. Leggett, Quantum Liquids (Oxford University Press, Oxford, 2006).

2 A. J. Leggett, Phys. Rev. Lett. 25, 1543 (1970).

3 A. J. Leggett, Phys. Fenn. 8, 125 (1973).

4 Y. Shi, Phys. Rev. B 72, 014533 (2005); erratum: Y. Shi, ibid. 74, 029901 (E) (2006). In the proof of nonexistence of ODLRO or NCRI there, we assumed that the localized single-particle wave function $\bar{w}(\mathbf{r})$ defined in an infinite interval satisfies $\bar{w}(\mathbf{r}) \bar{w}(\mathbf{r}-\mathbf{S}) \approx \bar{w}^{2}(\mathbf{r}) \exp (-|\mathbf{S}| / c)$. The proof can still go through if this condition is modified to another form such as $\bar{w}(\mathbf{r}) \bar{w}(\mathbf{r}-\mathbf{S}) \leq \bar{w}^{2}(\mathbf{r}) \exp (-|\mathbf{S}| / c)$ or $\bar{w}(\mathbf{r}) \bar{w}(\mathbf{r}-\mathbf{S})<q \exp (-|\mathbf{S}| / c)$, where $q$ is a constant of or less than the order of 1 . Both relations are satisfied by the Gaussian wave function.

5 E. Kim and M. H. W. Chan, Nature (London) 427, 225 (2004); E. Kim and M. H. W. Chan, Science 305, 1941 (2004).

6 A. S. C. Rittner and J. Reppy, Phys. Rev. Lett. 97, 165301 (2006).

7 Y. Aoki, J. C. Graves and H. Kojima, Phys. Rev. Lett. 99, 015301 (2007).

8 A. Penzev, Y. Yasuta and M. Kubota, J. Low Temp.Phys. 148, 677 (2007).

9 M. Kondo, S. Takada, Y. Shibayama, K. Shirahama, J. Low Temp. Phys. 148, 695 (2007).

10 B. Hunt, E. Pratt, V. Gadagkar, M. Yamashita, A. V. Balatsky, and J. C. Davis, Science 324, 632 (2009).

11 X. Lin, A. C. Clark and M. H. W. Chan, Nature 449, 1025
(2007); X. Lin, A. C. Clark, Z. G. Cheng, and M. H. W. Chan, Phys. Rev. Lett. 102, 125302 (2008).

12 J. Day, J. Beamish, J. Low Temp. Phys. 148, 683 (2007).

13 S. Sasaki, R. Ishiguro, F. Caupin, H. J. Maris, S. Balibar1, Science 313, 1098 (2006).

14 S. Sasaki, F. Caupin and S. Balibar, Phys. Rev. Lett. 99, 205302 (2007).

15 M. W. Ray, R. B. Hallock, Phys. Rev. Lett. 100, 235301 (2008).

16 J. Day, J. Beamish, Nature 450, 853 (2007).

17 J. T. West, O. Syshchenko, J. Beamish and M. H. W. Chan, Nature Phys. 5, 598 (2009).

18 A. F. Andreev and I. M. Lifshitz, Sov. Phys. JETP 29, 1107 (1969).

19 G. V. Chester, Phys. Rev. A 2, 256 (1970).

20 X. Dai, M. Ma, F. C. Zhang, Phys. Rev. B 72, 132504 (2005)

21 D. M. Ceperley and B. Bernu, Phys. Rev. Lett. 93, 155303 (2004); B. K. Clark and D. M. Ceperley, Phys. Rev. Lett. 96, 105302 (2006).

22 N. Prokof'ev and B. Svistunov, Phys. Rev. Lett. 94, 155302 (2005).

23 H. Matsuda and T. Tsuneto, Prog. Theor. Phys. Supp. 46, 411 (1970).

${ }^{24}$ Y. Imry and M. Schwartz, J. Low. Temp. Phys. 21, 543 (1975).

25 M. Schwartz, Phys. Rev. B 12, 3725 (1975).

26 M. Rossi J. Low. Temp. Phys. 153, 250 (2008).

27 D. E. Galli and L. Reatto, Phys. Rev. Lett. 96, 165301 
(2006).

28 C. Cazorla, G. E. Astrakharchik, J. Casulleras, and J. Boronat, New J. Phys. 11, 013047 (2009).

29 M. Boninsegni, A. B. Kuklov, L. Pollet, N.V. Prokofév, B.V. Svistunov and M. Troyer Phys. Rev. Lett. 97, 080401 (2006).

30 P. N. Ma, L. Pollet, M. Troyer and F. C. Zhang, J. Low Temp. Phys. 152, 156 (2008).

31 N. Prokof'ev, Adv. Phys. 56, 381 (2007).

32 G. D. Mahan and H. Shin, Phys. Rev. B 74, 214502 (2006).

33 A. S. C. Rittner and J. Reppy, Phys. Rev. Lett. 98, 175302 (2007).

34 E. Kim et al., Phys. Rev. Lett. 100, 065301 (2008).

35 S. Balibar and F. Caupin, J. Phys.: Condens. Matter 20, 173201 (2008).

36 A. C. Clark, J. T. West and M. H. W. Chan, Phys. Rev. Lett. 99, 135302 (2007).

37 M. Boninsegni, N. Prokofév and B. Svistunov, Phys. Rev. Lett. 96, 105301 (2006); L. Pollet, M. Boninsegni, A. B. Kuklov, N. V. Prokofév, B. V. Svistunov and M. Troyer, Phys. Rev. Lett. 98, 135301 (2007); S. G. Söyler, A.B. Kuklov, L. Pollet, N.V. Prokofév and B.V. Svistunov, arXiv.0908.4296.
38 D. Huse and Z. U. Khandker, Phys. Rev. B 75, 212504 (2007).

39 J. Toner, Phys. Rev. Lett. 100, 035302 (2008).

40 J. Wu and P. Phillips, Phys. Rev. B 78, 014515 (2008)

41 G. Biroli, C. Chamon and F. Zamponi, Phys. Rev. B 78, 224306 (2008)

42 A. V. Balatsky, M. J. Graf, Z. Nussinov, and S. A. Trugman, Phys. Rev. B 75, 094201 (2007); Z. Nussinov, A. V. Balatsky, M. J. Graf, and S. A. Trugman, Phys. Rev. B 76, 014530 (2007).

43 A. F. Andreev, JETP 108, 1157 (2009).

44 D. E. Galli and L. Reatto, J. Phys. Soc. Jpn. 77, 111010 (2008).

45 L. Pollet et al., Phys. Rev. Lett. 101, 097202 (2008).

46 P. W. Anderson, Science 324, 631 (2009).

47 H. Zhai and Y. S. Wu, J. Stat. Mech. P07003 (2005).

48 L. H. Nosanow and G. L. Shaw, Phys. Rev. 128, 546 (1962).

49 H. R. Glyde, Excitations in Liquid and Solid Helium (Clarendon Press, Oxford, 1994).

50 Any desent handbook of mathematics. 